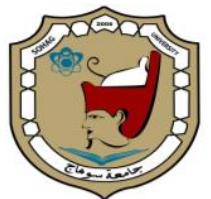

Sohag University

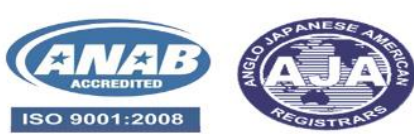

Sohag Medical Journal

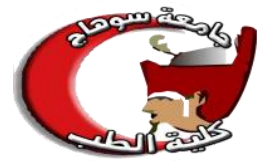

Faculty of Medicin

\title{
Impact of type 2 diabetes mellitus on cirrhotic patients
}

\author{
Sara Kasem Abdelaal. Ali Taha Ali . Ashraf A Askar
}

Department of Internal Medicine, Sohag Faculty of Medicine, Sohag University,

\begin{abstract}
:
Diabetes is frequent among cirrhotic patients. Hepatic encephalopathy and hepatocellular carcinoma, ascites, renal dysfunction, and bacterial infections are all possible complications. Diabetes is hypothesized to play a part in the advancement of fibrosis and cirrhosis, Additionally to NAFLD, by regulating multiple critical fibrogenesis mechanisms. Hyperglycemia is a significant predictor marker in people with cirrhosis, indicating an increased chance of death. When diabetes mellitus (DM) is first identified, its treatment should be incorporated into the patient's entire treatment plan. To reduce the danger of liver failure-related consequences and slow the advancement of chronic liver disease. End-stage renal disease is a frequent consequence of patients with liver cirrhosis, which is correlated to raised mortality and morbidity. patients with preexisting DM have a roughly doubled danger of hepatocellular carcinoma and HCC-related death. whatever the cause of the underlying CLD, HCC has a terrible prognosis, with a median survival of months.
\end{abstract}

Keywords: Diabetes mellitus, renal failure, liver cirrhosis, hepatic encephalopathy.

\section{Introduction:}

Diabetes is common among cirrhotic patients. Hepatic encephalopathy and hepatocellular carcinoma, ascites, renal dysfunction, and bacterial infections are all possible complications. ${ }^{(1)}$

Hyperglycemia is a significant predicttor marker in people with cirrhosis, indicating an increased chance of death. Diabetes is hypothesized to play a part in the advancement of fibrosis and cirrhosis, additionally to NAFLD, by regulating multiple critical fibrogenesis mechanisms ${ }^{(2)}$ End-stage renal disease is a frequent co-nsequence of patients with liver cirr-hosis, which is correlated to raised mo-rtality and morbidity. (3), Acute kidney injury affects about 20$50 \%$ among hospitalized patients with cirrhosis. ${ }^{(4)}$ A typical pathway of renal failure is active renal vasoconstriction, which can happen even in the early stages of the disease when routine renal function te-sts are normal. ${ }^{(5)}$

Diabetes has a negative prognostic effect in cirrhotic patients:-

\section{- Ascites and renal dysfunction}

In patients with cirrhosis caused by hepatitis $\mathrm{C}$, we have discovered that hyperglycemia is linked to the occurrence of ascites regardless of MELD score. (6) Unfortunately, the underlying mechanisms are still unknown.

Furthermore, diabetic nephropathy is frequently detected when kidney biopsy is conducted in cirrhotic patients on the waiting list for liver transplantation and it is indicative of the evolution of renal dysfunction following liver transplantation $^{(7)}$ 
- Hepatic encephalopathyDiabetes has been linked to numerable pathways that could cause hepatic encephalopathy. For starters, diabetes may boost ammonia generation by increasing type $\mathrm{K}$ glutaminase in the small intestine. Metformin, which decreases glutaminase activity in vitro, has been demonstrated to lessen the incidence of cirrhosis-related hepatic encephalopathy ${ }^{(8)}$. Second, the inflammatory condition linked to insulin resistance and type 2 diabetes could interact with cirrhosis and endotoxemia, both of which are linked to encephalopathy ${ }^{(9)}$. Third, as part of autonomic neuropathy in diabetes patients, intestinal motility impairment has been documented. It may encourage bacterial overgrowth in the small intestine, leading to bacterial translocation, which has been linked to hepatic encephalopathy ${ }^{(10)}$

\section{- Bacterial infections}

Diabetes and cirrhosis are two diseases that make you more susceptible to bacterial infections. ${ }^{(11)}$. A reduced T-cell-mediated immunological response could explain this trait in part. ${ }^{(12)}$ Diabetic patients have an abnormal neutrophil function ${ }^{(11)}$

\section{- Hepatocellular carcinoma}

patients with preexisting DM have a roughly doubled danger of hepatocellular carcinoma and HCC-related death $^{(13) .}$ Surprisingly, cirrhosis is only found in 50\% of NAFLD-rela-ted HCC patients ${ }^{(14)}$ It's possible that the process of hepato-carcinogenesis in metabolic syndrome is distinct from the conventional mechanism in cirrhosis Hyperinsulinemia, by upre-gulating growth signaling pathways and fostering an inflammatory environment, is thought to be important to the pathophysiological effects of diabetes on HCC formation. promoting angiogenesis and activating hepatic progenitor ${ }^{(15)}$ whatever the cause of the underlying CLD, HCC has a terrible prognosis, with a median survival of months. ${ }^{(16)}$ There is evidence that diabetic patients undergoing trans-arterial chemoembolization (TACE) are at an elevated danger for hepatic decompensation, liver abscess formation, and prolonged acute renal failure ${ }^{(17)}$.

\section{Diagnosis of diabetes in cirrhotic patients}

Diagnosing diabetes in cirrhotic patients can be difficult. First, Fasting serum glucose concentration is normal in 23 percent of persons with diabetes at an early stage, but serum glucose levels after a meal are more than $200 \mathrm{mg} / \mathrm{L}^{(18)}$

\section{TRADITIONAL GLYCEMIC MA- RKERS FOR cirrhotic PATIENTS}

It's fair to believe that early detection and starting therapy of DM in CLD patients would be advantageous

\section{- Fasting plasma glucose}

The best FPG cutoff for diagnosing $\mathrm{DM}$ in CLD/ESLD patients is still up for debate According to certain studies, the sensitivity of FPG in diagnosing pre-diabetes and DM in highrisk individuals was as low as $28.9 \%$ and 55.7 percent, respectively, when utilizing thresholds of 100-125 $\mathrm{mg} / \mathrm{dL}$ and $126 \mathrm{mg} / \mathrm{dL}^{(19)}$

\section{- Oral glucose tolerance test}

To diagnose diabetes, the OGTT is widely recommended. It's the best way to find gestational diabetes, cystic fibrosis-related diabetes, and post-transplant diabetes ${ }^{(20)}$

\section{- Hemoglobin A1c}

Because it reflects the average blood glucose for months, HbA1c is especially valuable as a marker for treatment efficacy While HbA1c rema-ins a helpful glycemic marker in the majority of patients with moderate liver 
disorders, its accuracy, and validity in patients with advanced liver diseases are still debated. The well-known curvilinear association between $\mathrm{HbA} 1 \mathrm{c}$ and erythrocyte turnover is to blame for $\mathrm{HbA1c}$ 's poor diagnostic performance. This might happen in CLD/ESLD patients due to hemorrhage caused by portal hypertension and coagulopathy. Splenomegaly causes hemolysis, whereas marrow suppression and nutritional insufficiency produce poor erythropoiesis. ${ }^{(21)}$

\section{- Glycated proteins}

GA and fructosamine are commonly utilized to detect glycemic control, during a length of time of two to three weeks. ${ }^{(22)}$ In individuals with chronic kidney disease and/or dialysis, GA has proven to be a particularly helpful glycemic measure. GA levels are unaffected by anemia, erythropoietin treatment, blood transfusion, or hemodialysis, unlike HbA1c. ${ }^{(23)}$ Disease conditions that disrupt protein metabolisms, predictably, harm GA and fructosamine accuracy ${ }^{(23)}$

\section{- Serum 1,5-anhydroglucitol}

The proximal renal tubules normally resorb the dietary monosaccharide 1,5anhydroglucitol (1,5-AG) in the blood. However, when it comes to hyperglycemia, glucosuria prevents reabsorption. In diabetic patients, and individuals at elevated danger of DM, serum 1,5-AG was discovered to be a day-today glycemic marker ${ }^{(24)}$. There is proof that in people with liver disorders, 1,5AG metabolism is disrupted ${ }^{(25)}$

\section{PATIENTS WITH LIVER DISEA- SES AND DIABETES: MANAGE- MENT}

The second problem in managing diabetes in patients with liver illnesses is to find a safe and efficient treatment plan for this medically complex population, particularly those with decompensated cirrhosis. Lifestyle modifications are widely acknowledged as essential in the medical therapy of both DM and liver disorders, When patients fail to achieve targeted glycemic control through lifestyle changes alone, antihyperglycemic medicines are often required

\section{- Metformin}

Metformin, which is typically used as a first-line oral medication for T2DM because of its effectiveness ${ }^{(26)}$ Several large retrospective investigations on diabetic people with CLD of diverse etiologies have shown that those treated with metformin have a 50\%-70 percent lower incidence of $\mathrm{HCC}^{(27)}$. Metformin has also been demonstrated to cut the danger of overt hepatic encephalopathy by 8 times by inhibiting glutaminase activity ${ }^{(28)}$. Despite its outstanding benefits in terms of morbidity and mortality, metformin is frequently withdrawn from patients with liver problems due to an overabundance of concern about metformin-associated lactic acidosis (MALA).

\section{- Pioglitazone}

Another extensively investigated antihyperglycemic drug that shows promise in the treatment of nonalcoholic steatohepatitis (NASH) is the thiazolidinedione pioglitazone. Potential troglitazone-related hepatotoxicity, rosiglitazone-related cardiovascular hazards, and pioglitazone-related bladder malignancy all lingering concerns about the longterm safety of thiazolidinedione medication remaining a roadblock to pioglitazone's broad usage in clinical practice (28)

\section{- $\alpha$-glucosidase inhibitors}

Even though $\boldsymbol{\alpha}$-glucosidase inhibitors like acarbose, voglibose, and miglitol have limited use in the general diabetic population because of GIT side effects such as flatulence and diarrhea, they deserve to be recognized as a potential antihyperglycemic treatment in patients 
with CLD. Acarbose medication has also been demonstrated to lower serum ammonia levels and improve cognitive perform-ance by favoring saccharolytic, rather than proteolytic, intestinal bacterial flora. It's a good way to treat hyperglycemia and mild hepatic encephalopathy all at the same time ${ }^{(29)}$

\section{- GLP-1 receptor agonists}

Because of their capacity to encourage weight reduction and lower the danger of hypoglycemia, GLP-1 receptor agonists such as exenatide and liraglutide are becoming a more prominent class of incretin-based medicine as a medication for T2DM. Several studies in diabetic patients with hepatic steatosis found reductions in hepatic steatosis, aminotransferase levels, and liver fibrosis score after therapy with liraglutide. ${ }^{(30)}$

\section{- DPP-4 inhibitors}

They are weight-neutral and have a low danger of hypoglycemia, similar to GLP-1 receptor agonists but they have the extra benefit of being oral. ${ }^{(31)}$

Except for vildagliptin, most DPP-4 inhibitors are deemed safe in individuals with mild or moderate hepatic impairment; nonetheless, due to limited researches, caution is indicated in patients with severe hepatic impairment (32)

\section{- Sodium-glucose cotransporter-2 inhibitors}

They have been shown to ameliorate hepatic steatosis and liver fibrosis in various animal models of NAFLD/NASH. ${ }^{(33)}$

Brief safety evidence on the use of SGLT-2 inhibitors in cirrhotic people is restricted due to a lack of therapeutic expertise. However, In patients with mild to marked liver injury, canagliflozin and ertugliflozin are usually regarded safe. ${ }^{(34)}$

Individuals with varying degrees of hepatic impairment can take empag- liflozin without changing their dosage. According to limited data, ${ }^{(35)}$ however dapagliflozin may require dose reduction in people with advanced hepatic disease $^{(36)}$

\section{- $\quad$ Sulfonylureas and meglitinides}

Because both sulfonylureas and meglitinides are substantially metabolized by the liver and are strongly bound to serum proteins, people with liver disease are more vulnerable due to lower drug inactivation and elevated free drug concentrations.

Specifically, meta-analyses of numerous case-control studies found that patients with T2DM who were treated with sulfonylureas had a threefold greater risk of developing HCC. Hyperinsulinemia could be to blame. ${ }^{(37)}$ Insulin secretagogues should be prevented or administered with extreme caution in individuals with CLD/ESLD, according to expert opinion ${ }^{(38)}$

\section{- Insulin}

Despite the growing number of antihyperglycemic drugs, insulin and insulin analogs remain the best and safest glycemic therapy for people with diabetes.

Even in individuals with decompensated cirrhosis and marked liver injury, they can be taken cautiously. (39) A primary limiting side effect is hypoglycemia. Cirrhosis, on the one hand, can cause or worsen hyperinsulinemia and insulin resistance, due to poor hepatic gluconeogenesis and sarcopenia, cirrhotic individuals may have an increased insulin response. ${ }^{(40)}$ These competing modes of action, together with the GIT symptoms that many CLD patients experiences, make it challenging to anticipate a patient's exogenous insulin requirement on a day-to-day basis.

Data from observational studies reveal a link between insulin therapy and the occurrence of $\mathrm{HCC}$ in people with 
SOHAG MEDICAL JOURNAL

Vol. 25 No. September 32021
T2DM, similar to the situation of sulfonylurea. ${ }^{(41)}$

\section{References}

1. Elkrief L, Rautou PE, Sarin S, Valla D, Paradis V, Moreau RJLI. Diabetes mellitus in patients with cirrhosis: clinical implications and management. 2016;36(7):936-48.

2. Chung W, Promrat K, Wands JJWJoH. Clinical implications, diagnosis, and management of diabetes in patients with chronic liver diseases. 2020;12(9):533.

3. Angeli $\mathrm{P}$, Ginès $\mathrm{P}$, Wong $\mathrm{F}$, Bernardi $\mathrm{M}$, Boyer TD, Gerbes A, et al. Diagnosis and management of acute kidney injury in patients with cirrhosis: revised consensus recommendations of the International Club of Ascites. 2015;64(4):531-7.

4. Huelin P, Piano S, Solà E, Stanco M, Solé $\mathrm{C}$, Moreira R, et al. Validation of a staging system for acute kidney injury in patients with cirrhosis and association with acuteon-chronic liver failure. 2017-\&rA:(r) lo . $₹ 0 \mathrm{e} 5$.

5. Nadim MK, Kellum JA, Davenport A, Wong F, Davis C, Pannu N, et al. Hepatorenal syndrome: the 8th international consensus conference of the Acute Dialysis Quality Initiative (ADQI) Group. 2012;16(1):1-17.

6. Elkrief L, Chouinard P, Bendersky N, Hajage D, Larroque B, Babany G, et al. Diabetes mellitus is an independent prognostic factor for major liver-related outcomes in patients with cirrhosis and chronic hepatitis C. 2014;60(3):823-31.

7. Calmus Y, Conti F, Cluzel P, Hill G, Antoine $\mathrm{C}$, Scatton $\mathrm{O}$, et al. Prospective assessment of renal histopathological lesions in patients with end-stage liver disease: effects on long-term renal function after liver transplantation. 2012;57(3):5726.

8. Ampuero J, Ranchal I, Nunez D, del Mar Díaz-Herrero M, Maraver M, Del Campo JA, et al. Metformin inhibits glutaminase activity and protects against hepatic encephalopathy. 2012;7(11):e49279.

9. Basu S, Zethelius B, Helmersson J, Berne C, Larsson A, Ärnlöv JJIjoc, et al. Cytokine-mediated inflammation is independently associated with insulin sensitivity measured by the euglycemic insulin clamp in a community-based cohort of elderly men. 2011;4(2):164.

10.Bauer TM, Schwacha H, Steinbrückner B, Brinkmann FE, Ditzen AK, Aponte JJ, et al. Small intestinal bacterial overgrowth in human cirrhosis is associated with systemic endotoxemia. 2002;97(9):2364-70.

11.Muller L, Gorter K, Hak E, Goudzwaard W, Schellevis F, Hoepelman A, et al. Increased risk of common infections in patients with type 1 and type 2 diabetes mellitus. 2005;41(3):281-8.

12.Pozzilli P, Leslie RJItodm. Infections, immunity, and diabetes. 2003.

13.Everett JAJPo. The 12 items social and economic conservatism scale (SECS). 2013;8(12):e82131.

14.Piscaglia F, Svegliati-Baroni G, Barchetti A ‘Pecorelli A, Marinelli S, Tiribelli C, et al. Clinical patterns of hepatocellular carcinoma in nonalcoholic fatty liver disease: a multicenter prospective study. 2016;63(3):827-38.

15.Bohinc BN, Diehl AMJCild. Mechanisms of disease progression in NASH :new paradigms. 2012;16(3):549-65.

16. Wang Y-G, Wang P, Wang B, Fu Z-J, Zhao W-J, Yan S-LJPo. Diabetes mellitus and poorer prognosis in hepatocellular carcinoma: a systematic review and metaanalysis. 2014;9(5):e95485.

17. Shin JU, Kim KM, Shin SW, Min SY ‘Park SU, Sinn DH, et al. A prediction model for liver abscess developing after transarterial chemoembolization in patients with hepatocellular carcinoma. 2014;46(9):8137. 
18. Moreau R, Delègue $\mathrm{P}$, Pessione F, Hillaire $S$, Durand F, Lebrec D, et al. Clinical characteristics and outcome of patients with cirrhosis and refractory ascites. 2004;24(5):457-64.

19.Kim KS, Kim SK, Lee YK, Park SW, Cho YWJDM. Diagnostic value of glycated hemoglobin (HbA1c) for the early detection of diabetes in high-risk subjects. 2008;25(8):997-1000.

20.Care AAJD. 2. Classification and diagnosis of diabetes: standards of medical care in diabetes-2018. 2018;41(Supplement 1):S13-S27.

21. Gonzalez-Casas R, Jones EA, Moreno-Otero RJWjogW. The Spectrum of anemia associated with chronic liver disease. 2009;15(37):4653.

22. Koga M, Kasayama SJEj. Clinical impact of glycated albumin as another glycemic control marker. 2010:1008090468.-

23.Freedman BI, Shenoy RN, Planer JA, Clay KD, Shihabi ZK, Burkart JM, et al. Comparison of glycated albumin and hemoglobin A1c concentrations in diabetic subjects on peritoneal and hemodialysis. 2010;30(1):72-9.

24.Carmody D, Naylor RN, Bell CD, Berry S, Montgomery JT, Tadie EC, et al. GCKMODY in the US National Monogenic Diabetes Registry: frequently misdiagnosed and unnecessarily treated. 2016;53(5):703-8.

25.Juraschek SP, Miller III ER, Appel LJ, Christenson RH, Sacks FM, Selvin EJDM. Effects of dietary carbohydrate on 1, 5anhydroglucitol in a population without diabetes: results from the OmniCarb trial. 2017;34(10):1407-13.

26.Klachko D, Whaley-Connell AJCm. Use of metformin in patients with kidney and cardiovascular diseases. 2011;1(2):87-95.

27.Lai S-W, Chen P-C, Liao K-F, Muo C-H, Lin C-C, Sung F-CJAJoG. Risk of hepatocellular carcinoma in diabetic patients and risk reduction associated with anti-diabetic therapy: a population-based cohort study. 2012;107(1):46-52.

28.Hampp C, Pippins JJP, safety d. Pioglitazone and bladder cancer: FDA's assessment. 2017;26(2):117-8.

29.Bredenoord AJ, Chial HJ, Camilleri M, Mullan BP, Murray JAJCG, Hepatology. Gastric accommodation and emptying in the evaluation of patients with upper gastrointestinal symptoms. 2003;1(4):26472.

30. Yan J, Yao B, Kuang H, Yang X, Huang Q, Hong $\mathrm{T}$, et al. Liraglutide, sitagliptin rand insulin glargine added to metformin: the effect on body weight and intrahepatic lipid in patients with type 2 diabetes mellitus and nonalcoholic fatty liver disease. 2019;69(6):2414-26.

31. Pratley RE, Salsali AJCmr, opinion. Inhibition of DPP-4: a new therapeutic approach for the treatment of type 2 diabetes. 2007;23(4):919-31.

32. Giorda CB, Nada E, Tartaglino BJE. Pharmacokinetics, safety, and efficacy of DPP-4 inhibitors and GLP-1 receptor agonists in patients with type 2 diabetes mellitus and renal or hepatic impairment. A systematic review of the literature. 2014;46(3):406-19.

33. Honda $\mathrm{Y}$, Imajo $\mathrm{K}$, Kato $\mathrm{T}$, Kessoku T, Ogawa Y, Tomeno W, et al. The selective SGLT2 inhibitor ipragliflozin has a therapeutic effect on nonalcoholic steatohepatitis in mice. 2016;11(1):e0146337.

34. Sahasrabudhe V, Terra SG, Hickman A, Saur D, Raje S, Shi H, et al. Pharmacokinetics of single-dose ertugliflozin in patients with hepatic impairment. 2018;40(10):1701-10.

35. Macha S, Rose P, Mattheus M, Cinca R, Pinnetti $S$, Broedl $U$, et al. Pharmacokinetics, safety and tolerability of empagliflozin, a sodium-glucose 
SOHAG MEDICAL JOURNAL

Vol. 25 No. September 32021
Impact of type 2 diabetes mellitus on cirrhotic patients Sara Kasem Abdelaal cotransporter 2 inhibitor, in patients with hepatic impairment. 2014;16(2):118-23.

36. Kasichayanula S, Liu X, Zhang W, Pfister M, LaCreta FP, Boulton DWJCt .Influence of hepatic impairment on the pharmacokinetics and safety profile of dapagliflozin: an open-label, parallelgroup, single-dose study. 2011;33(11):1798-808.

37.Lee J-Y, Jang S-Y, Nam CM, Kang ESJSr. Incident hepatocellular carcinoma risk in patients treated with a sulfonylurea: a nationwide, nested, case-control study. 2019;9(1):1-6.

38.Gangopadhyay KK, Singh PJIjoe, metabolism. Consensus statement on dose modifications of antidiabetic agents in patients with hepatic impairment. 2017;21(2):341.

39.Kupčová V, Arold G, Roepstorff C, Højbjerre M, Klim S, Haahr HJCdi. Insulin degludec: pharmacokinetic properties in subjects with hepatic impairment. 2014;34(2):127-33.

40.Periyalwar P, Dasarathy SJCild. Malnutrition in cirrhosis: contribution and consequences of sarcopenia on metabolic and clinical responses. 2012;16(1):95-131.

41.Hassan MM, Curley SA, Li D, Kaseb A, Davila M, Abdalla EK, et al. Association of diabetes duration and diabetes treatment with the risk of hepatocellular carcinoma. 2010-116(8): 46-1938. 